\title{
Correction to: Paraquat-Induced Movement Disorder in Relation to Oxidative Stress-Mediated Neurodegeneration in the Brain of Drosophila melanogaster
}

\author{
S. Niveditha ${ }^{1}$ - S. R. Ramesh ${ }^{1} \cdot$ T. Shivanandappa ${ }^{1}$ \\ Published online: 9 January 2018 \\ ๑) Springer Science+Business Media, LLC, part of Springer Nature 2018
}

\section{Correction to: \\ Neurochemical Research (2017) 42:3310-3320 \\ https://doi.org/10.1007/s11064-017-2373-y}

The original version of this article unfortunately contained a mistake. The entries missing in the reference list are given below and their corresponding citations are provided in the Discussion section text.

There have been conflicting reports on the effect of acute dose treatment of PQ on the activity of antioxidant enzymes in Drosophila. In some of the earlier studies, acute exposure to PQ led to increased activity of SOD $[17,35]$, whereas, others have reported reduced activity of SOD at higher dose [37, 38]. In our study, flies exposed to multiple-dose of PQ showed a markedly decreased activity of both $\mathrm{Cu} / \mathrm{Zn}-\mathrm{SOD}$ and mitochondrial Mn-SOD in contrast to the acute exposure. Catalase, on the other hand, was increased by both single and multiple dose exposures. Increased SOD activity in the acute dose model suggests a transient adaptive response of antioxidant defense mechanism against PQ-induced oxidative stress which could be reversible [17]. However, the lasting effect of the decreased activity of SOD in flies exposed to the multiple dose of PQ indicates irreversible deterioration of the antioxidant defense [39], which leads to sustained oxidative stress. The

The original article can be found online at https://doi.org/10.1007/ s11064-017-2373-y.

T. Shivanandappa

tshivanandappa@yahoo.com

1 Neurobiology Laboratory, Department of Zoology, University of Mysore, Manasagangotri, Mysore,

Karnataka 570006, India activity of AChE, a general biochemical marker of neural function, involved in cholinergic neurotransmission was significantly increased in both acute and multiple dose of PQ treatments, suggesting a biochemical response to a neurotoxic compound and the state of increased oxidative stress. However, the degree of increased AChE activity in flies exposed to multiple dose of PQ was lower compared to that of acute exposure. The reduction of AChE activity observed in the multiple dose treatment could be attributed to cholinergic dysfunction and degeneration, which is considered as an important contributor to PD symptoms [40, 41].

Maintaining a healthy population of mitochondria in a cell is an essential process in view of their role in energy production in the form of ATP. Mitochondrial damage is considered as one of the key abnormalities that are causally linked to neurodegeneration $[14,42,43]$. Pesticides implicated in the pathogenesis of neurodegenerative disease are known to cause mitochondrial abnormalities [44].

\section{References}

37. Krůček T, Korandová M, Šerý M et al (2015) Effect of low doses of herbicide paraquat on antioxidant defense in Drosophila. Arch Insect Biochem Physiol 88:235-248

38. Shukla AK, Ratnasekhar C, Pragya P et al (2016) Metabolomic analysis provides insights on paraquatinduced Parkinson-like symptoms in Drosophila melanogaster. Mol Neurobiol 53:254-269

39. Bostantjopoulou S, Kyriazis G, Katsarou Z et al (1996) Superoxide dismutase activity in early and advanced Parkinson's disease. Funct Neurol 12:63-68 
40. Shimada H, Hirano S, Shinotoh H et al (2009) Mapping of brain acetylcholinesterase alterations in Lewy body disease by PET. Neurology 73:273-278

41. Müller MLTM., Bohnen NI (2013) Cholinergic dysfunction in Parkinson's disease. Curr Neurol Neurosci Rep 13:377
42. Beal MF (2004) Mitochondrial dysfunction and oxidative damage in Alzheimer's and Parkinson's diseases and coenzyme Q 10 as a potential treatment. J Bioenerg Biomembr 36:381-386 